February 1995

rev. May 1995

IFP-713-UNC

\title{
Quark Mass Textures within a Finite Non-abelian Dicyclic Group.
}

\author{
Paul H. Frampton and Otto C. W. Kong \\ Institute of Field Physics, Department of Physics and Astronomy, \\ University of North Carolina, Chapel Hill, NC 27599-3255
}

\begin{abstract}
Using as a flavor symmetry a finite nonabelian dicyclic $Q_{2 N}$ group, we show how to derive quark mass matrices with two arrangements of symmetric texture zeros which are phenomenologically viable. Three other such acceptable textures in the recent literature are unattainable in this approach and hence disfavored. We assume massive vector-like fermions and Higgs singlets transforming as judiciously-chosen $Q_{2 N}$ doublets and use the tree-level mass generation mechanism of Froggatt and Nielsen.
\end{abstract}

Typeset using REVTEX 
One of the longest standing problems in particle theory has been the values of the fermion masses. Aside from the compelling classification of quarks and leptons into families, based on masses and quark mixing angles, the masses themselves remain a cunundrum. For example, the mass hierarchy: why is the top quark over a third of a million times heavier than the electron?

There has recently been considerable interest in the structure of the quark mass matrices, particularly in the idea of postulating texture zeros in grand unified theories [1], [2], [3] with a view to obtaining relations between the masses and mixing angles. A list of phenomenologically viable quark mass matrices bearing a maximum number of symmetric texture zeros was presented in 4 . The possibility of constructing such mass matrices from a scheme of gauged flavor symmetry has been considered in [5] and [6].

There has also been considerable activity in the use of finite non-abelian groups as flavor symmetries [7], 8], [9], with a view to generating the mass hierarchy.

Here we attempt a synthesis of these two approaches and construct desirable quark mass matrix textures by using a nonabelian flavor symmetry (specifically a dicyclic group $Q_{2 N}$ ) together with the Froggatt-Nielsen mechanism of mass generation [10]. While U(1) flavor symmetry constructions for quark mass matrices with nonsymmetric hierarchical textures have been attempted [11], the full list of such phenomenologically viable quark mass matrices is not yet available. Our approach does not a priori give a symmetric texture. However, as a first attempt, we consider here only the possibility of constructing the symmetric texture patterns presented in [4]. The general case of non-symmetric textures would naturally be a very interesting next step.

The use of $Q_{2 N}$ as a finite flavor group has been discussed in detail in [9]. We recall here that the irreducible representations of $Q_{2 N}$ are four singlets $1,1^{\prime}, 1^{\prime \prime}, 1^{\prime \prime \prime}$ and $(N-1)$ doublets $2_{(k)}$, with $1 \leq k \leq(N-1)$. Most important for our purposes are the products:

$$
2_{(k)} \times 2_{(l)}=2_{(|k-l|)}+2_{(\min \{k+l, 2 N-k-l\})}
$$

where, in a generalized notation, $2_{(0)} \equiv 1+1^{\prime}$ and $2_{(N)} \equiv 1^{\prime \prime}+1^{\prime \prime \prime}$. 
We assign the quarks to $Q_{2 N}$ representations as follows:

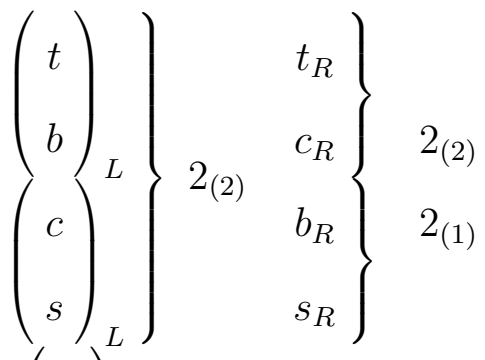

$$
\begin{aligned}
& \left(\begin{array}{l}
u \\
d
\end{array}\right)_{L} \begin{array}{llll}
1^{\prime} & u_{R} & 1^{\prime} \\
& & d_{R} & 1
\end{array}
\end{aligned}
$$

When we embed the finite spinorial $Q_{2 N}$ into its continuous progenitor $S U(2), 1,2_{(1)}$ and $\left(1^{\prime}+2_{(2)}\right)$ correspond respectively to the singlet, doublet and triplet representations. The above quark assignment is thus anomaly-free if the leptons are assigned to:

$$
\begin{aligned}
& \left.\begin{array}{c}
\left(\begin{array}{c}
\nu_{\tau} \\
\tau^{-}
\end{array}\right)_{L} \\
\nu_{\mu} \\
\mu^{-}
\end{array}\right)_{L} \\
& \left.\left(\begin{array}{c}
\nu_{e} \\
e^{-}
\end{array}\right)_{L} \begin{array}{lll}
\tau_{L}^{+} \\
& & \mu_{L}^{+}
\end{array}\right\} 2_{(2)}
\end{aligned}
$$

We shall not consider lepton masses further here. For the mass textures of the quarks we postulate heavy vector-like fermions and singlet Higgs and assume the quark masses arise from tree graphs as in [10].

As the first of two successful examples, we demonstrate how to derive the five-zero texture in Eqs. (2) and (3) below. [Note that no texture with the maximum number of six texture zeros can be phenomenolically viable [4].]

$$
M_{u}=\left(\begin{array}{ccc}
0 & 0 & \lambda^{4} \\
0 & \lambda^{4} & \lambda^{2} \\
\lambda^{4} & \lambda^{2} & 1
\end{array}\right)
$$




$$
M_{d}=\left(\begin{array}{ccc}
0 & \lambda^{4} & 0 \\
\lambda^{4} & \lambda^{3} & 0 \\
0 & 0 & 1
\end{array}\right)
$$

For these matrices we have suppressed all coefficients of order one since at the present stage we are satisfied to derive only the correct orders in $\lambda$ for each entry.

The standard Higgs scalar doublets of $S U(2)_{L}$ are taken as a $2_{(4)}$ of $Q_{2 N}$ coupling to the up quarks, and a $2_{(3)}$ coupling to the down quarks. We assume these get VEVS that break $Q_{2 N}$ and give mass only to the third family. For the up quark mass matrix the entry $\left(M_{u}\right)_{33}$ is of order 1 from the coupling $t_{L}\left(2_{(2)}\right) t_{R}\left(2_{(2)}\right) H_{u}\left(2_{(4)}\right)$. At leading order, all other entries $\left(M_{u}\right)_{i j}$ vanish. Similarly, $b_{L}\left(2_{(2)}\right) b_{R}\left(2_{(1)}\right) H_{d}\left(2_{(3)}\right)$ gives $\left(M_{d}\right)_{33}$ of order 1 and no other $\left(M_{d}\right)_{i j}$

To obtain the other entries in Eqs. (2) and (3) at order $\lambda^{n}\left(\lambda \sim \sin \theta_{C} \sim 0.22\right.$ where $\theta_{C}$ is the Cabibbo angle), we introduce a list of vector-like quark doublets $Q_{i}\left(2_{(i)}\right)(i=$ $6,7,10,13,14)$, singlets $U_{i}\left(2_{(i)}\right)(i=6,10,14)$ and $D_{i}\left(2_{(i)}\right)(i=4,17)$, bearing the same standard model quantum numbers as $Q_{L}, u_{R}$ and $d_{R}$ respectively, together with standard model singlet Higgses $S_{i}\left(2_{(i)}\right),(i=5,8,13,14,17,20)$. Although this set of $Q_{2 N}$ doublets seems long and $a d$ hoc, it is highly constrained (see below). Since we have assumed heavy particles in doublets up to $2_{20}$ the flavor group, of order 84 , is $Q_{42}$.

We choose a set of bases and label the two states in the heavy fermion $Q_{2 N}$ doublets as $2_{(i)+}$ and $2_{(i)-}$, which lie respectively in the third and second family direction. The $H_{u} \mathrm{VEV}$ then allows only the six couplings:

$$
\begin{gathered}
t_{L}<H_{u}>U_{6+} ; Q_{6+}<H_{u}>t_{R} ; Q_{6+}<H_{u}>U_{10+} ; \\
Q_{10+}<H_{u}>U_{6+} ; Q_{10+}<H_{u}>U_{14+} ; Q_{14+}<H_{u}>U_{10+} .
\end{gathered}
$$

and the $H_{d}$ VEV only the two couplings:

$$
Q_{7+}<H_{d}>D_{4+} ; Q_{14+}<H_{d}>D_{17+}
$$


The $S_{i}$ VEVS may then be chosen to give certain vertices such as : $U_{6+}^{\dagger}<S_{8+-}>c_{R}$, $U_{10+}^{\dagger}<S_{8+-}>c_{R}$, and others. We define:

$$
\begin{gathered}
\lambda^{2}=\frac{<S_{i}>}{M_{\text {even }}} \\
\lambda=\frac{<S_{i}>}{M_{\text {odd }}}
\end{gathered}
$$

where $M_{\text {even }}$ and $M_{\text {odd }}$ denote the mass of a heavy fermion in $Q_{2 N}$ representation $2_{(k)}$ for being $k$ even and odd respectively. Note that Eqs. (4) and (5) are acceptable because the k-even and k-odd doublets occur independently in the irreducible representations of the covering $S U(2)$ in the sense that the k-even doublets appear only in vectors of $\mathrm{SU}(2)$ and the k-odd doublets appear only in spinors.

We have now all the ingredients of the model. In the low energy efffective field theory, after integrating out the heavy fermions [12], we have the tree level quark mass matrices having the structure of the model denoted by roman numeral $\mathrm{V}$ in ref. [四], namely those exhibited in Eqs.(2) and (3) above.

For instance, the $\left(M_{u}\right)_{32}$ entry is given by the Froggatt-Nielsen tree graph (shown in Fig.(1a)) correponding to the operator couplings

$$
t_{L}<H_{u}>\left(U_{6+} U_{6+}^{\dagger}\right)<S_{8+-}>c_{R}=\lambda^{2}<H_{u}>t_{L} c_{R} ;
$$

while $\left(M_{u}\right)_{13}$ is given by the graph of Fig. (1c) corresponding to:

$$
\begin{gathered}
t_{L}<H_{u}>\left(U_{6+} U_{6+}^{\dagger}\right)<S_{8+-}>\left(U_{14-} U_{14-}^{\dagger}\right)<S_{14-}>u_{R} \\
=\lambda^{4}<H_{u}>t_{L} u_{R} ;
\end{gathered}
$$

and $\left(M_{d}\right)_{22}$ is given by Fig. (2a) corresponding to:

$$
\begin{gathered}
s_{L}<S_{5+-}>\left(Q_{7+}^{\dagger} Q_{7+}\right)<H_{d}>\left(D_{4+} D_{4+}^{\dagger}\right)<S_{5+-}>s_{R} \\
=\lambda^{3}<H_{d}>s_{L} s_{R} .
\end{gathered}
$$


The other entries in $M_{u}$ and $M_{d}$ are derived similarly; some further examples are shown in Fig. (1b) for $M_{u}$ and Figs. (2b) and (2c) for $M_{d}$.

In the construction of the model, we followed a systematic procedure and were surprised to realize that it is highly non-trivial if any consistent model can be constructed at all. The difficulty is not only to derive the correct texture zeros but also to avoid unwanted entries at too low an order in $\lambda$. We find only two consistent models, the above model and one alternative summarized below.

The mass matrices for the alternative model have texture structures of the model denoted by roman numeral IV in ref. «1. They are:

$$
\begin{gathered}
M_{u}=\left(\begin{array}{ccc}
0 & \lambda^{6} & 0 \\
\lambda^{6} & \lambda^{4} & \lambda^{2} \\
0 & \lambda^{2} & 1
\end{array}\right) \\
M_{d}=\left(\begin{array}{ccc}
0 & \lambda^{4} & 0 \\
\lambda^{4} & \lambda^{3} & 0 \\
0 & 0 & 1
\end{array}\right)
\end{gathered}
$$

Note that the $M_{d}$ matrix is the same as in our first example but $M_{u}$ is changed; as before, we neglect coefficients of order unity. The $Q_{2 N}$ assignments for the quarks and leptons are the same as they were previously.

The entries in Eqs. (6) and (7) at order $\lambda^{n}$ are constructed, as in the previous example, through introducing vector-like $Q_{2 N}$ quark doublets $Q_{i}\left(2_{(i)}\right)(i=6,7,10,18,23)$, singlets $U_{i}\left(2_{(i)}\right)(i=6,10,18)$ and $D_{i}\left(2_{(i)}\right)(i=4,11)$, together with standard model singlet Higgses $S_{i}\left(2_{(i)}\right),(i=5,8,11,15,16,18,23)$. As mentioned in our first example this set of doublets which seems long and $a d$ hoc is really highly constrained. For this second example, the flavor group is $Q_{48}$.

Under the same kind of state labelling, the $H_{u}$ VEV then allows only the four couplings:

$$
t_{L}<H_{u}>U_{6+}, Q_{6+}<H_{u}>t_{R}
$$




$$
Q_{6+}<H_{u}>U_{10+}, Q_{10+}<H_{u}>U_{6+}
$$

and the $H_{d} \mathrm{VEV}$ only the coupling:

$$
Q_{7+}<H_{d}>D_{4+}
$$

The $S_{i}$ VEVS may then be chosen to give certain vertices such as : $U_{6+}^{\dagger}<S_{8+-}>c_{R}$, $U_{10+}^{\dagger}<S_{8+-}>c_{R}$, and so on.

The general procedure is as follows: the quarks and leptons has to come from $S U(2)_{H}$ singlets, doublets and triplets. There are only 21 anomaly free schemes of assignment with no extra chiral fermions [13]. We are aiming at assignments that can lead to up- and downquark mass matrices with different hierarchical textures [4]. That leaves us with two schemes of which only the one used here gives interesting models.

Picking the above scheme, the feasiblity of using the Froggatt-Nielsen mechanism enforces the first family quarks to be $Q_{2 N}$ singlets. We then introduce appropriate heavy fermions and Higges whenever necessary as we go on to build entries of higher order in $\lambda$, keeping track of overall consistency.

Attempts to construct models giving texture models I, II, and III of ref. [4] lead to conflicts, and we therefore conclude that those patterns of texture zeros are disfavored.

In this approach, the standard model gauge group $G=S U(3)_{C} \times S U(2)_{L} \times U(1)_{Y}$ is extended to $G \times\left(Q_{2 N}\right)_{\text {global }}$ which, in turn, is assumed a subgroup of $G \times S U(2)_{H}$ where $S U(2)_{H}$ is a gauged horizontal symmetry. This last point is important because the imposition of the necessary anomaly cancellation restricts the assignment of the quarks and leptons to $Q_{2 N}$ representations as discussed above.

The authors of [4] have analyzed all possible symmetric quark mass matrices with the maximal (six) and next-to-maximal (five) number of texture zeros, and concluded that only five models, denoted by the roman numerals I to $\mathrm{V}$ in their work, are phenomenologically viable. By insisting on derivation of the texture zeros from the $Q_{2 N}$ dicyclic flavor symmetry, we have reduced the number of candidates to two, denoted in [4] by the roman numerals IV and V. 
It seems likely that similar considerations could be made directly at the $S U(5) \times Q_{2 N}$ level because the assignment of the quarks and leptons are consistent with an $S U(5)$ embedding, and this - together with the generalization to supersymmetry - are presently under investigation.

In conclusion, the reduction in the number of free parameters in the low energy theory attained by postulating texture zeros in the fermion mass matrices has been shown to have a dual description in terms of a horizontal symmetry $Q_{2 N} \subset S U(2)_{H}$. This $S U(2)_{H}$ could arise in a GUT group or directly from a superstring. Our main point is that the derivation of the values of the fermion masses and quark mixings in a putative theory of everything may likely involve a horizontal symmetry, probably gauged, as an important intermediate step. The two simple cases given in the present paper illustrate how this can happen.

This work was supported in part by the U.S. Department of Energy under Grant DEFG05-85ER-40219, Task B.

We thank an anonymous referee for helping to clarify the presentation.

\section{Figure Captions.}

Fig.1 Froggatt-Nielsen tree graphs for $M_{u}$. (The symmetric counterpart $\left(M_{u}\right)_{23}$, and second graphs for $\left(M_{u}\right)_{22}$ and $\left(M_{u}\right)_{33}$ are not shown).

Fig.2 Froggatt-Nielsen tree graphs for $M_{d}$. 


\section{REFERENCES}

[1] S. Dimopoulos, L. J. Hall and S. Raby, Phys. Rev. Lett. 68, 1984 (1992); Phys. Rev. D45, 4195 (1992); ibid. D46, R4793 (1992);

G. Anderson, S. Raby, S. Dimopoulos, and L. J. Hall, ibid D47, 3702 (1993)

[2] G.F. Giudice, Mod. Phys. Lett. A7, 2429 (1992).

[3] G.K. Leontaris and N.D. Tracas, Phys. Lett. B303, 50 (1993);

G.K. Leontaris and J.D. Vergados, ibid. B305, 242 (1993).

[4] P. Ramond, R.G. Roberts and G.G. Ross, Nucl. Phys. 406, 19 (1993).

[5] E. Papageorgiu, Z. Phys. C64, 509 (1994).

[6] L. Ibanez and G. G. Ross, Phys. Lett. B332, 100 (1994).

[7] S. Pakvasa and H. Sugawara, Phys. Lett. 73B, 61 (1978); ibid. 82B, 105 (1979).

[8] D.B. Kaplan and M. Schmaltz, Phys. Rev. D49, 3741 (1994).

[9] P. H. Frampton and T. W. Kephart, Phys. Rev. D51, R1 (1995), and UNC-Chapel Hill Report IFP-702-UNC.

[10] C. D. Froggatt and H. B. Nielsen, Nucl. Phys. B147, 277 (1979).

[11] M. Leurer, Y. Nir and N. Seiberg, Nucl. Phys.B398, 319 (1993); ibid. B420, 468 (1994); E. Dudas, S. Pokorski and C.A. Savoy, Yukawa Matrices from a Spontaneously Broken Abelian Symmetry. preprint SPhT Saclay T95/027, MPI-PTh 95-33

[12] H. Georgi, Nucl. Phys. B (Proc. Suppl.) 29B,C, 1 (1992).

[13] D.S. Shaw and R.R. Volkas, Phys. Rev. D47, 241 (1993). 


\section{FIGURES}

$(a):\left(M_{u}\right)_{32}$

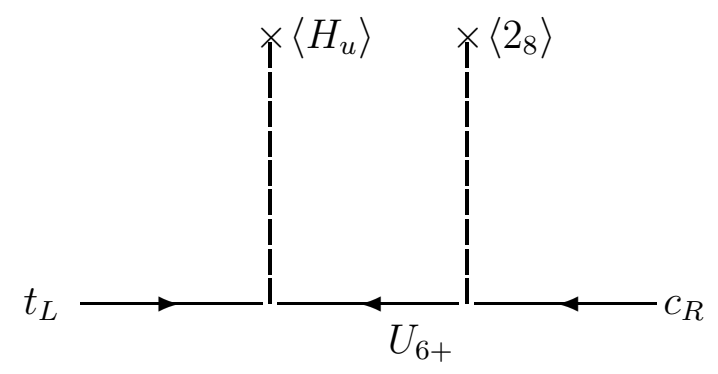

$(b):\left(M_{u}\right)_{22}$

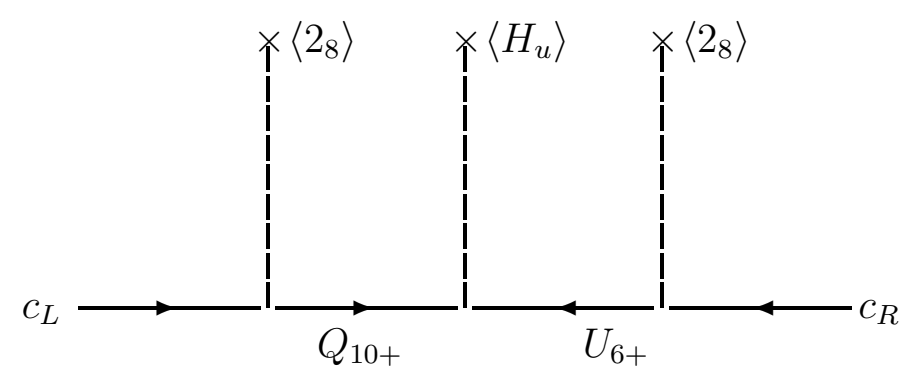

$(c):\left(M_{u}\right)_{13}$

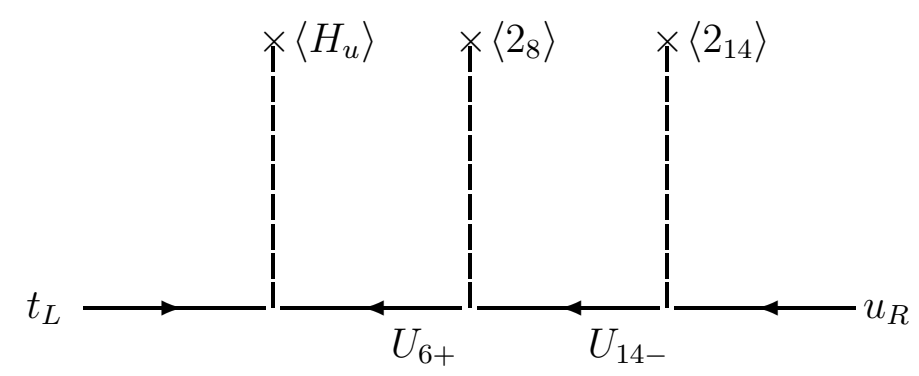

FIG. 1. Froggatt-Nielsen tree graphs for $M_{u}$. (The symmetric counterpart $\left(M_{u}\right)_{23}$, and second graphs for $\left(M_{u}\right)_{22}$ and $\left(M_{u}\right)_{31}$ are not shown) 

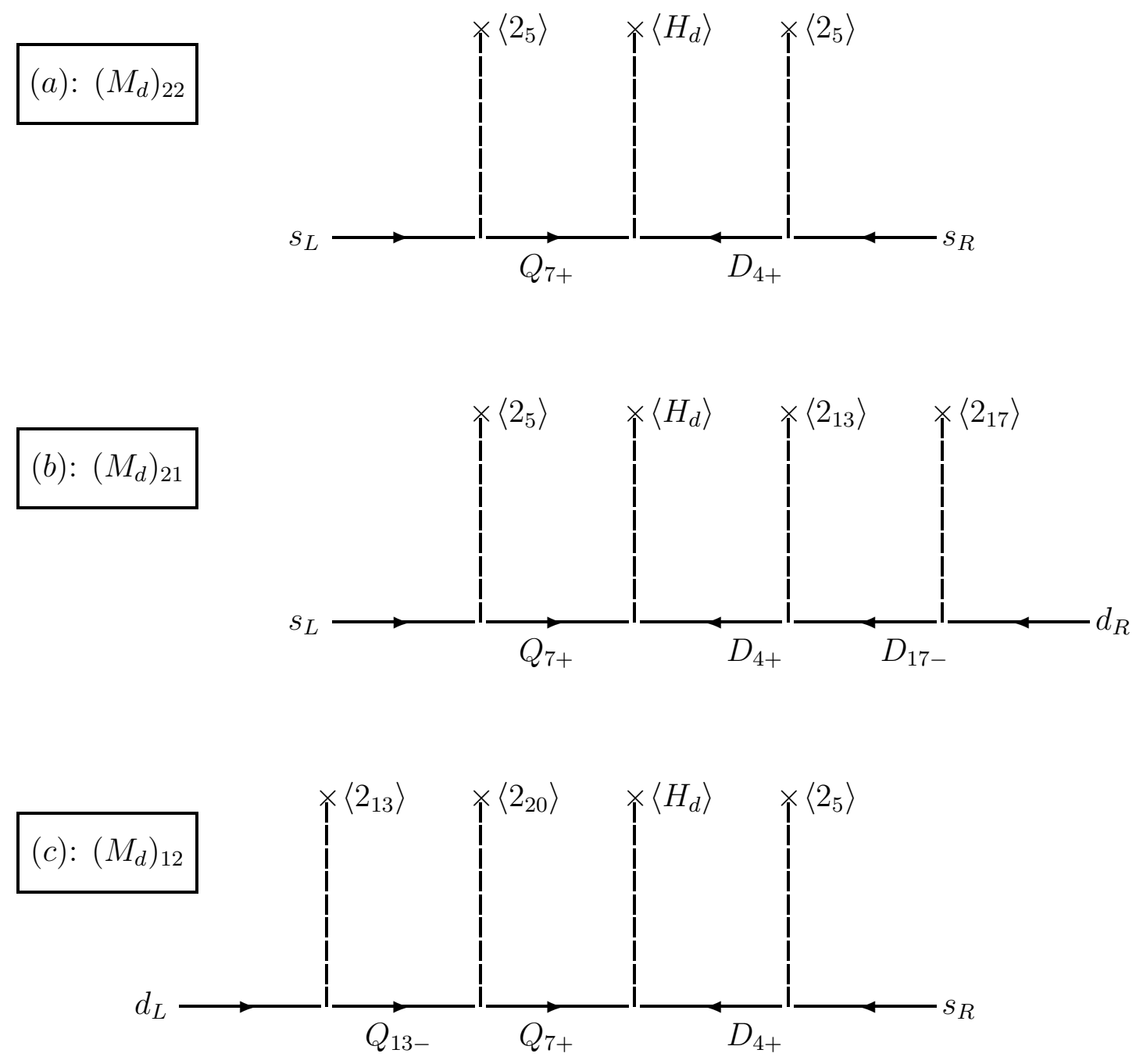

FIG. 2. Froggatt-Nielsen tree graphs for $M_{d}$. 